\title{
Indagine preliminare della natura fisica del fenomeno che ha originato il periodo sismico irpino dell'Agosto 1962
}

\author{
D. Di Filippo - F. Peronaci
}

Ricevuto il 23 Marzo 1963

\begin{abstract}
Rrassunto. - Si riportano i risultati di uno studio microsisunico esegruito sulle scosse più intense, che hanno caratterizzato il periodo sismico dell'Irpinia dell'Agosto 1962. Esegnita la deteminazione dei dati ipocentrali si fa osservare che lipocentro ha una profondita superiore (40 kin circa) di quella riscontrata a oggri negli studi di terrenoti appenninici. La delerminazione della natura fisica della scossa all'ipocentro ha portato ad uno schema di frattura il eui piano si accorda con la direzione delle faglie profonde che interessano l'Italia Meridionale con un andamento trasversale rispetto alla dorsale Appenninica.
\end{abstract}

SUMMARY. .... The paper reports the results of a microseismical study carried out on the most intense quakes which characterized the seismic period of August 1962 in Irpinia. After determining the data, the anthor points ont low the focus is situated deeper than found in any other study on earthquakes in the Appennines (40 km). The detemination of the physical nature of the quake at its focus has lead to a fracture scheme which has a plan in accordance with the direction of the deep fauls interesting sontheru Italy across the Appentine ridge.

\section{1 - CONSIDERAZIONI GENERALI.}

Nel 1962 la regione Irpina è stata ancom una volta colpita da uma serie di fenomeni sismici, che a partire dal 21 Igosto si sono protratti per vari giorni, dando luogo ad un periodo sismico che deve considerarsi tra i più intensi di quelli verificati nella stessa zona negli ultimi decemin. L'area macrosismica comprende le provincie di Avellino, Benevento, Salerno, Napoli, Caserta, Potenza, Bari, Chieti, Campobasso e nella zona di massima intensità (IX grado) sono compresi i paesi di Ariano Irpino, S. Areangelo Trimonte, Molinara, Buonalbergo, Apice. A Napoli la scossa più intensa è stata avvertita di $V$, a Roma di III. 
Ia tabella 1 comprende l'elenco delle scosse delle quali sono state determinate la magnitudo e la relativa energia; in particolare i dati delle prime quattro scosse sono stati ottenuti con le registrazioni della stazione di Padova, gli altri sono stati calcolati implegando i sismogrammi di Roma.

Tabella 1

\begin{tabular}{|c|c|c|c|c|}
\hline Giorno & $\begin{array}{c}\text { Ora } \\
\text { a }\end{array}$ & $\begin{array}{l}\text { di reg. } \\
\text { Roma }\end{array}$ & Magnitudo & $\begin{array}{c}\text { Energia } \\
\text { (erg.) }\end{array}$ \\
\hline 21-8 & $15^{\mathrm{h}} 5$ & $56^{\mathrm{m}} 22^{\mathrm{s}}$ & 4,52 & $7,05 \cdot 10^{18}$ \\
\hline$n$ & 180 & $08 \quad 24$ & 4,38 & $3,65 \cdot 10^{18}$ \\
\hline$n$ & 180 & $09 \quad 43,5$ & 5,86 & $5,38 \cdot 10^{21}$ \\
\hline$"$ & 182 & $20 \quad 05,5$ & 6,18 & $2,61 \mid 10^{2}$ \\
\hline$"$ & 184 & $45 \quad 29$ & 4,49 & $9,33 \cdot 10^{18}$ \\
\hline$"$ & 200 & $08 \quad 57$ & 4,05 & $7,03 \cdot 10^{17}$ \\
\hline$"$ & $21 \quad 2$ & $28 \quad 35,5$ & 3,94 & $4,00 \cdot 10^{17}$ \\
\hline$n$ & $23 \quad 3$ & $36 \quad 37,5$ & 3,56 & $6.21 \cdot 10^{16}$ \\
\hline$n$ & $23+$ & $40 \quad 57$ & 3,46 & $3,88 \cdot 10^{16}$ \\
\hline $22-8$ & 020 & $05 \quad 25,5$ & 3,36 & $2,27 \cdot \mid\left(0^{10}\right.$ \\
\hline
\end{tabular}

In Fig. 1 è rappresentato l'andamento del periodo sismico a partire dalle ore 10 del 21 Agosto.

Il fatto che la zona, particolarmente interessante dal punto di vista sismo-tettonico, non sia mai stata oggetto di uno studio microsisnico particolareggiato, nonché la sua posizione, tra il basso Tirreno e le Alpi Dinariche, che rappresentano regioni da noi già studiate, ci banno indotto ad intraprendere uno studio delle scosse più intense di questo periodo sismico.

Un primo esame delle registrazioni gentilmente inviateci dagli osservatori a cui le avevamo richieste, ci ha presentato un quadro notevolmente complesso delle sollecitazioni verificatesi: nelle stazioni vicine ad esempio la scossa delle $18^{\mathrm{n}} 10^{\mathrm{m}}$ ca. ha inizio mentre è in atto la registrazione di una scossa di intensità più modesta, mentre nei sismogrammi delle stazioni a maggiore distanza quella delle $18^{\mathrm{n}} 21^{\mathrm{m}}$ ea. ha inizio mentre è registrata la coda della scossa delle $18^{\mathrm{h}} 10^{\mathrm{m}}$. 
Ia deteminazione microsismica dei dati ipocentrali quindi presentava notevoli difficolta per la individuazione degli inizi. Questi fatti ci hamno eonsigliato di affrontare il problema esaninando prima la scossa delle ore $15^{\mathrm{h}} 56^{\mathrm{m}}$ che è stata registrata dalle stazioni vicine (Napoli, Roma, Rocea di Papa, Aquila, Tamanto) e quindi ottenuti per essa

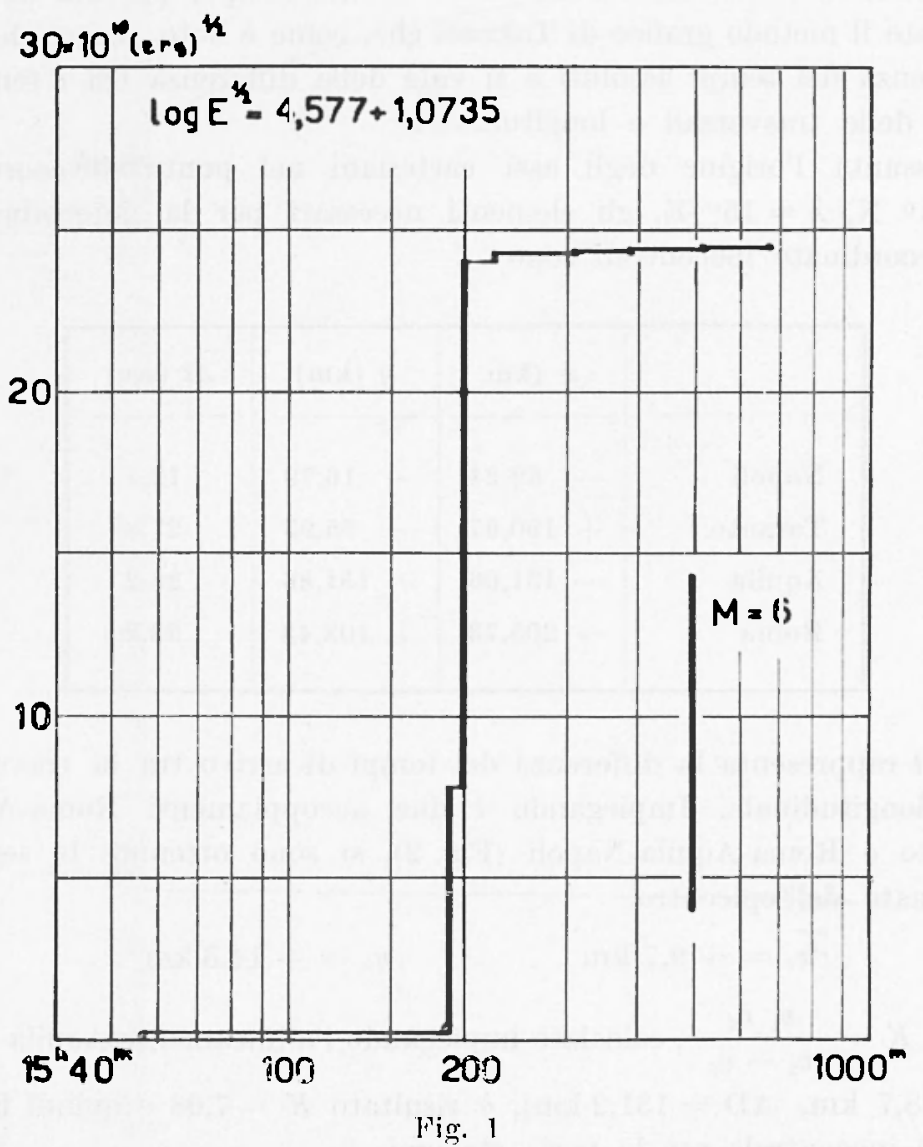

i dati ipocentrali (da noi assunti come provvisori), passare a determinare con un metorlo statistico le coordinate dell'ipocentro e l'ora origine delle due scosse più intense. Questa volta le stazioni impiegate sono sufficientemente lontane onde ridure al minimo l'influenza delle stratificazioni superficiali sulla velocita di propagazione delle onde sismiche. Sono stati quindi utilizzati i dati degli osservatori con distanze epicentrali comprese tra 500 e $1.800 \mathrm{~km}$. 


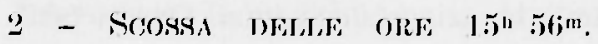

la miglioni regristmazioni di fuessla scossa sono quolle ottenute a Napoli, Aquila, Tamanto, Roma; in particolare la stazione di Tamanto

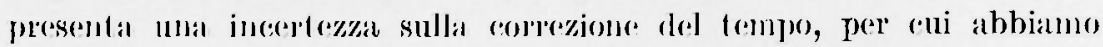
applicato il metodo grafico di Takabsi che, come è noto, presceinde dalla conoseonzal dei tempi assoluli a si vale della diflerenzal tra i tempi di arrivo dolle trasversali a longritudimali.

Assunta l'origine degli assi artessiani nel punto di coondinate $\varphi=110 \mathrm{~N}, \hat{\lambda}-150 \mathrm{E}$, gli clementi necessieri jer la determinazione dello roomdinate iporentrali somo

\begin{tabular}{|c|c|c|c|}
\hline & $x(\mathrm{k} m$ & $y(k+1)$ & $a t$ (ser) \\
\hline Niapoli & -62.24 & $-\quad 16,79$ & 12.1 \\
\hline ' l'aralulo & $+190,97$ & - 55,93 & 27,8 \\
\hline Aquila & $-131,06 \mathrm{j}$ & 151,86 & 28,2 \\
\hline Roma & $-205,73$ & $+\quad 103,43$ & 33,2 \\
\hline
\end{tabular}

Ove It mpenerenta la differenza dee tempi di arrivo tra la trasversale - la longitudinale. Impiegando i due areoppiamenti Roma-AquilaTaranto a Roma-A(puila-Napoli (Fig. 2), si sono ottenute le seguenti coordinale dell'epirentro

$$
r_{o}=-1 \cdot 9,7 \mathrm{~km} \quad \quad y_{0}=+14,3 \mathrm{~km} .
$$

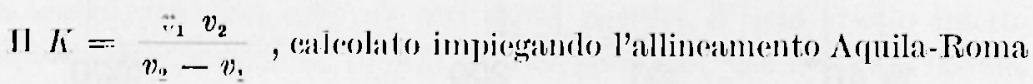
$(a=88,7 \mathrm{~km}, \Delta D=131,2 \mathrm{~km})$, ̀̀ risultato $K=7,08$ e quindi la profondita ipoecentrale per le varie stazioni

$$
\begin{aligned}
& \text { Napoli } 35,5 \mathrm{~km} \\
& \text { Aruila } 32,5 \\
& \text { Tamanto } 31,5 .
\end{aligned}
$$

La nostua preferenza ra al valore ottenuto ron la stazione di Napoli che is la pin vicina all'ipoeentro $\left(\Lambda_{i p .}=85,7 \mathrm{~km}\right)$. Quindi in coordinate geografiche i dali relativi alla scossa sono

$$
r_{\circ}=11^{\circ} 07^{\prime} 43^{\prime \prime} \mathrm{N} ; \quad \lambda_{0} \cdots 15^{\circ} 06^{\prime} 57^{\prime \prime} \mathrm{E} ; \quad h=35,5 \mathrm{~km} \text {. }
$$


D. DI FILIPPO - F. PERONACI

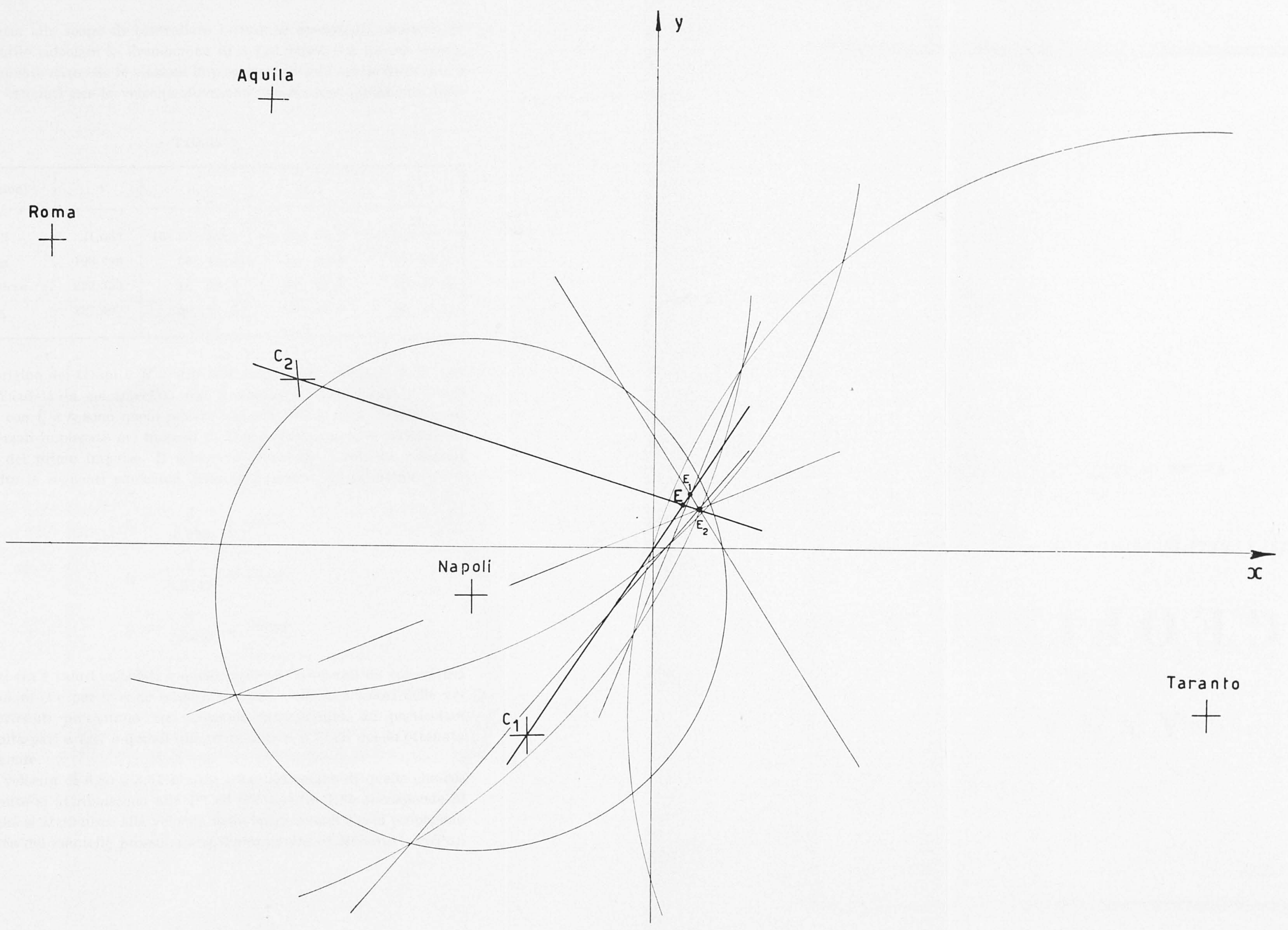

Fig. 2 
Anche allo scopo di controllare i risultati precedenti abbiamo ritenuto utile calcolare le dromocrone di 3 fasi relative a questa scossa. Naturalmente dato che le stazioni impiegate sono solo quelle della tab. 2 i valori ottenuti per le velocita dovranno considemarsi puramente indi(ativi.

Tabella 2

\begin{tabular}{|c|c|c|c|c|c|c|}
\hline Stazioni & $\Delta$ & 1 & & $t_{2}$ & $t_{3}$ & \\
\hline Napoli & 81,667 & $1.5^{11} 55^{\mathrm{m}}$ & $58 \mathrm{~s}, 8$ & $15^{11} 56^{\mathrm{m}} 10^{\mathrm{s}}, 9$ & $15^{11} 55^{\mathrm{m}}$ & 575,7 \\
\hline Aquila & 198,889 & 56 & 15,4 & $56 \quad 43,6$ & 56 & 12,2 \\
\hline l'eschiera & 223,333 & 56 & 20,0 & $56 \quad 52,0$ & 56 & 16.0 \\
\hline Roma & 237,407 & 56 & 21,6 & $56 \quad 54,8$ & 56 & 17,5 \\
\hline
\end{tabular}

L'origine dei tempi is $H=15^{\mathrm{n}} 55^{\mathrm{m}} 46^{\mathrm{s}}, 2$ quale proviene dalla curva di Waadati da noi tracciata con le stazioni a disposizione. I tempi indicati con $t_{1}$ e $t_{2}$ sono quelli relativi all'arrivo delle onde longitudinali e trasversali impiegate nel metodo di Takansi, mentre $t_{3}$ si riferisce all'arrivo del primo impulso. Il calcolo eseguito con i minimi quadlati lar fornito le seguenti equazioni delle dromocrone pin probabili

$$
\begin{aligned}
& t_{1}=\frac{\Delta}{6,005}+0,4975 \\
& t_{2}=\frac{\Delta}{3,5147}+1,3730 \\
& t_{3}=\frac{\Delta}{7,8430}+1,0209
\end{aligned}
$$

gli scarti tra i valori calcolati a quelli registrati sono tali da consentirei di affermare che pur avendo impiegato poche stazioni $i$ valori delle velocita ottenuti presentano una notevole attendibilita. In particolare il $K$ risulta pari a 7,27 e quindi differente di solo 0,19 da quello ottenuto graficamente.

Ise velocità di 6,80 e $3,51 \mathrm{~km} / \mathrm{sec}$ sono dell'ordine di quelle che eomumemente si attribuiscono alle $\mathrm{P}^{*}$ ed $\mathrm{S}^{*}$, mentre 7,84 corrisponde al valore che si attribuisee alla velocità delle longitudinali che si propagano nella zona del mantello prossima alla discontinuità di Nohorovieic (Pn). 
Il primo impulso a Napoli $(1=81,667 \mathrm{~km})$ è una Pn e non uns Py (o P*) come avviene nomalmente per tale distanza e terremoti superficiali. Tale fatto è spiegabile solo quando si attribuisca ma conveniente profondita al terremoto e contemporaneamente si consideri lo

\begin{tabular}{|c|c|c|c|}
\hline & $\Delta t_{1}$ & $A I_{2}$ & $\Delta t_{3}$ \\
\hline Napoli & - $0^{5}, 093$ & $\ldots, 0,02$ & $-0,060 ;$ \\
\hline Aquila & 0,545 & $\quad 0,559$ & $+0,379$ \\
\hline Peschiera & $\ldots 0,461$ & $-0,886$ & $-0,304$ \\
\hline Roma & $\therefore \quad 0.0009$ & $\div \quad 0,318$ & $+0,009$ \\
\hline
\end{tabular}

strato del granito di potenza ridotta. Questo trova conferma nel nostro caso, nella natura vulcanica della zona, dalle osservazioni geologiche che damno il basalto affiomente ad esempio a Ustica, nonché da studi precedenti nei quali si è riscontrato dhe il primo strato della crosta si assottiglia verso l'Italia meridionale. Infine il fatto che la Pll preceda la $P^{*}$ nella registrazione di Napoli, fa sospettare che almeno in tale direzione lo spessore della crosta terestre sia inferiore a quello at tribuito per l'Italia centrale.

Tutti questi fatti ei hanno indotto a nom impiegare le stazioni vicine per la deteminazione dei dati ipocentrali relativi alle seosse piu intense, utilizzando invece gli elementi che provengono da registrazioni di stazioni più lontane onde ridure al minimo l'influenza delle anomalie regionali.

\section{3 - SCOSSA DELLE ORE $18^{\mathrm{n}} 10^{\mathrm{m}}$ ("a.}

Nelle stazioni vicine l'inizio di questa seossa è registrato mentre avveniva la registrazione di un terremoto proveniente dalla stessa zona ipocentrale e di intensità noterolmente inferiore. S a questo riguardo caratteristica la registrazione di Napoli ove appare al completo la registrazione della prima scossa, a quindi l'inizio della seconda che ha dato origine al salto delle pemnine. Analogo fatto si è osservato nei sismogrammi di Roma e Aquila per quanto riguarda i fotosismografi. L'inizio della scossa piu intensa appare in ogni caso non disturbato e 
perfottamente individuabile in tutte le stazioni a distanza epicentrale superiore ai $500 \mathrm{~km}$.

Impiegando il metodo statistico di Caloi-Peronaci abbiamo determinato i dati microsismici della scossa. Al riguardo abbiamo assunto come epicentro provvisorio quello ottennto precedentemente, assegnando alla profondita $u$ valore provvisorio di $40 \mathrm{~km}$ onde poter più agevolmente interpolare le due dromocrone di Jeffreys per $h=33 \mathrm{~km}$ e $h=96,4 \mathrm{~km}$. La tabella 3 riassume gli elementi necessari per il calcolo statistico, quale proviene dall'aver applicato alle stazioni l'equazione di condizione:

$$
\begin{aligned}
\delta t_{o}-\cos \varphi_{o} \operatorname{sen} a \frac{\partial f}{\partial 1} \delta \lambda_{o} & -\cos \alpha \frac{\partial f}{\partial j} \delta \phi_{o}+\frac{\partial f}{\partial h} \delta h_{o}=T_{o}+ \\
& -\left[t_{o}+f\left(\varphi_{o}, \lambda_{o}, h_{o}\right)\right]
\end{aligned}
$$

ove $\alpha$ è l'azimut della stazione rispotto all'epicentro, $f\left(\varphi_{0}, \lambda_{o}, h_{0}\right)$ il tempo di propagazione della longitudinale per $h=40 \mathrm{~km}, T_{o}$ il tempo desunto dal sismogramma, $t_{o}$ il tempo origine provvisorio, $\frac{\partial f}{\partial t}$ l'incremento di tempo calcolato sulla dromocrona per $\Delta=1^{\circ}$ ed infine $\frac{\partial f}{\partial t}$ la variazione del tempo di tragitto per un ammento di profondità pari a $63,4 \mathrm{~km}$.

L'applicazione del metodo di Gauss ha condotto ai seguenti risultati che rappresentano nelle unità indicate, le correzioni più probabili da apportare ai valori provvisori delle incognite

$$
\begin{aligned}
& \delta h_{o}=+0,1490946 \pm 0,2858331 \\
& \delta \varphi_{o}=+0,0427733 \pm 0,0500885 \\
& \delta \lambda_{o}=-0,0008224 \pm 0,0281936 \\
& \delta t_{o}=+0,6759963 \pm 1,8624467
\end{aligned}
$$

L'errore medio dell'unità di peso è $\varepsilon= \pm 0,39221024$ con $[l l 4]=$ 1,3844615 e $[v v]=1,3844599$.

te coordinate dell'ipocentro a il tempo origine sono quindi

$$
\begin{aligned}
& \phi_{o}=41^{\circ} 10^{\prime} 16^{\prime \prime} \mathrm{N} \pm 0^{\circ} 03^{\prime} 00^{\prime \prime}, 5 \\
& \lambda_{o}=15^{\circ} 06^{\prime} 57^{\prime \prime} \mathrm{E} \pm 0^{\circ} 01^{\prime} 41^{\prime \prime}, 5 \\
& h_{o}=49,45 \mathrm{~km} \pm 18,11 \\
& H_{o}=18^{\mathrm{n}} 09^{\mathrm{m}} 03^{\mathrm{s}} \pm 1^{\mathrm{s}}, 86 .
\end{aligned}
$$




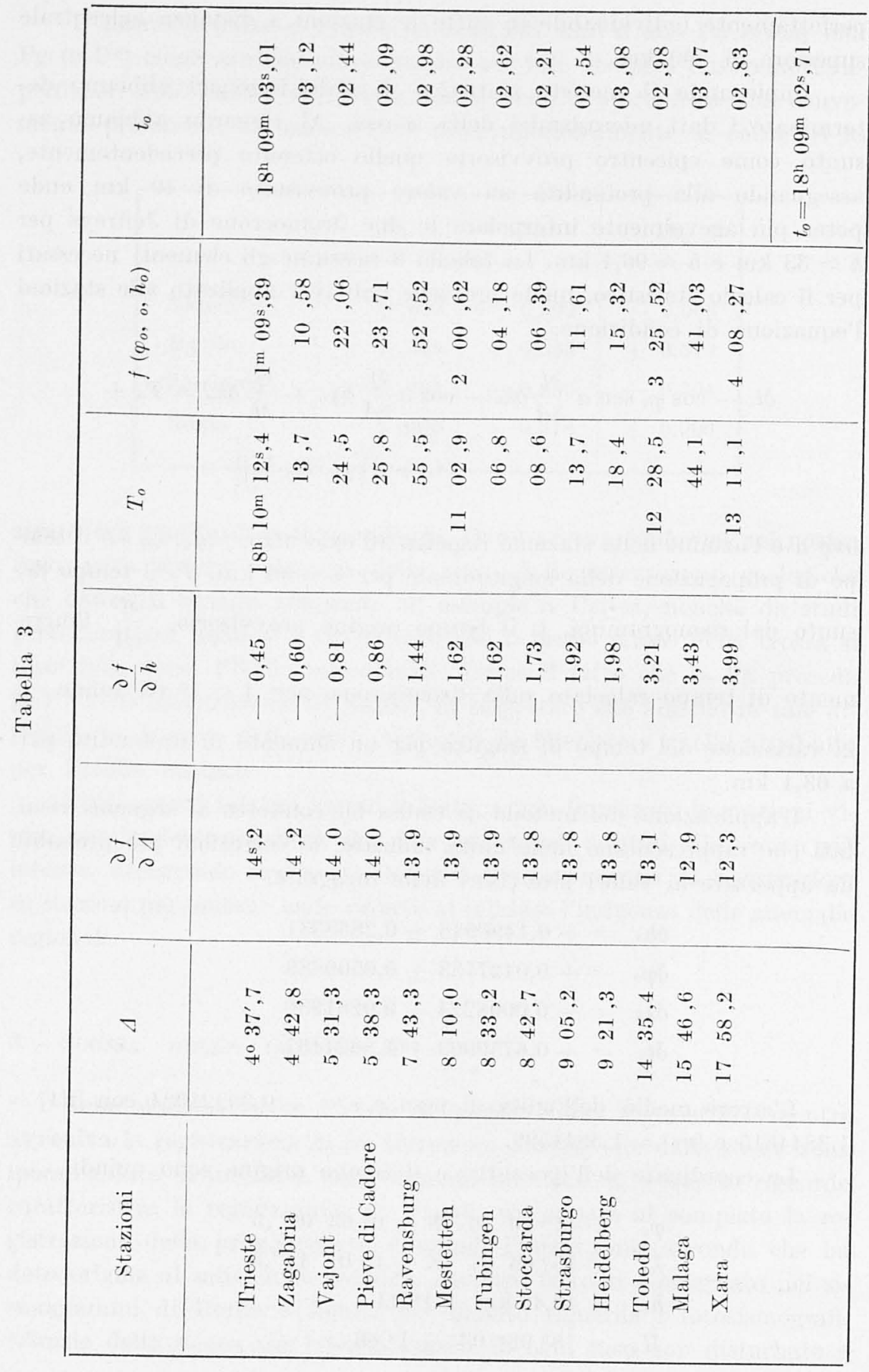


Si noterà che gli errori probabili superano le correzioni da apportare ai valori provvisori per eni non si ritiene utile una ulteriore approssimazione. L'errore probabile sulla profondità ipocentrale trova una giustificazione non solo nel fatto che tale grandezza presenta sempre in ogni sua determinazione una notevole incertezza, ma anche nel fat to che, come vedremo, l'ipocentro trovasi in una zona di frattura. Inoltre la natura della regione, tomentata dal punto di vista geologico, e la successione senza intervallo delle scosse nelle registrazioni sono altrettanti elementi che influiscono negativamente in una determinazione dei dati ipocentrali. Accetteremo i risultati ottenuti come definitivi.

In ogni caso la profondità del fuoco sismico è superiore a quella che si è riscontrata in tutti i terremoti studiati per l'Appennino centrosettentrionale, e ciò fa pensare che la zona che è oggetto dello studio rappresenti l'inizio o giù di li della regione ove si sono riscontrati terremoti a profondità intermedia, legati al rulcanismo attivo del Basso Tirreno.

4 - SCOSSA DEIJIE ORE $18^{\mathrm{n}} 19^{\mathrm{m}}$ cal.

La sua magnitudo è 6,18 ed è la più intensa dell'intero periodo sismico. La tab. 4 riporta gli elementi impiegati nella determinazione dei dati ipocentrali calcolati sempre a partire dai valori provvisori:

$$
p_{o}=41^{\circ} 07^{\prime}, 7 \mathrm{~N} ; \quad \lambda_{o}=15^{\circ} 07^{\prime}, 0 \mathrm{E} ; \quad h_{0}=40 \mathrm{~km} \text {. }
$$

Il tempo origine provvisorio $H_{o}=18^{\mathrm{h}} 19^{\mathrm{m}} 29^{\mathrm{s}}, 17$ è stato ottenuto come media dei tempi origine nelle singole stazioni quali provengono dalla dromocrona di Jeffreys.

I risultati ottenuti sempre con lo stesso metodo statistico sono:

$$
\begin{aligned}
& \delta h_{o}=-0,0037636 \pm 0,2347430 \\
& \delta \varphi_{0}=+0,0332820 \pm 0,0335391 \\
& \delta \lambda_{o}=-0,0432276 \pm 0,0 \tilde{5} 94 \tilde{5} 43 \\
& \delta t_{o}=+0, \tilde{a} \tilde{5} 32033 \pm 0,6790361 \\
& \varepsilon= \pm 0,260323 ; \quad[l l .4]=0,54214257 ; \quad[v v]=0, \check{0} 4214256 \text {. }
\end{aligned}
$$

I valori più probabili delle coordinate ipocentrali e del tempo origine sono quindi:

$$
\begin{aligned}
& \phi_{o}=41^{\circ} 09^{\prime} 41^{\prime \prime}, 8 \mathrm{~N} \pm 0^{\circ} 02^{\prime} 00^{\prime \prime}, 7 \\
& \lambda_{o}=5^{\circ} 04^{\prime} 24^{\prime \prime}, 2 \mathrm{E} \pm 0^{\circ} 03^{\prime} 34^{\prime \prime}, 0 \\
& h_{o}=39,76 \mathrm{~km} \pm 14,88 \\
& H_{o}=18^{\prime \prime} 19^{\mathrm{m}} 29^{\mathrm{s}}, 62 \pm 0^{\mathrm{s}}, 68 .
\end{aligned}
$$


Tabella 4

\begin{tabular}{|c|c|c|c|c|c|c|}
\hline Stazioni & & $d$ & $T_{o}$ & & $h_{o}$ & \\
\hline Trieste & & $37^{\prime}, 7$ & $18^{n} 20 \mathrm{~m}$ & $38^{*}, 8$ & $18^{11} \quad 9^{1 \mathrm{~m}}$ & $29 \mathrm{~s}, 41$ \\
\hline Kalgaloria & & 42,8 & & 39.9 & & 29.32 \\
\hline Vajont & & 51,3 & & 50.9 & & $2 x, 8 t$ \\
\hline Ravenshurer & & 43,3 & 21 & 22.1 & & 29.58 \\
\hline Westetten & & 18,0 & & 29,7 & & 29,08 \\
\hline T'ubinghen & & 33,2 & & 33,7 & & 29.52 \\
\hline Stoconarda & & $+2,7$ & & 35,4 & & 29,01 \\
\hline Strashurgo & 9 & $0.5,2$ & & 40,5 & & 28,89 \\
\hline Il eidelbergr & & 21.25 & & $4+, 6$ & & 29,28 \\
\hline Clermont $F$. & & 51,4 & & 51,5 & & 29.20 \\
\hline Toledo & 14 & 35,4 & 22 & 54.9 & & 29.38 \\
\hline \multirow[t]{2}{*}{ Malara } & & 46.6 & 23 & 10,5 & & 29,47 \\
\hline & & & & & \multicolumn{2}{|c|}{$t_{0}=1811199^{m} 295,17$} \\
\hline
\end{tabular}

$\check{\jmath}$ - NATURA Fisich DELLE SCOSSE.

Ina delle ragioni che ci ha indotto ad eseguire lo studio del periodo sismico irpino ì stata quella di inquadrare il terremoto nel complesso dei fenomeni sismo-tettonici che interessano l'Italia e le regioni linitrofe, portando un ulteriore contributo agli studi da noi rompiuti a questo riguardo. In particolare nel nostro caso, la zona dell'Irpinia presenta un grande interesse in quanto trovasi tra il Tirreno meridionale a le Alpi Dinariche che furono oggetto di analoghe trattazioni. Ci siano quindi proposti di determinare, impiegando gli impulsi iniziali, la natura fisica delle due scosse più intense. A questo riguardo, dato il tipo di registrazioni ottenute, abbiano ritenuto necessatrio calcolare le dromocrone più probabili dei due prini impulsi registrati per ognuma delle due scosse. Implegando i dati di tab. 5 relativi a stazioni comprese tra 500 a $1.600 \mathrm{~km}$, onde poter approssimare le dromocrome a rette, per quanto si riferiscer alla scossa delle $18^{n} 09^{m} 033^{*}, 39$, la dromocrona 


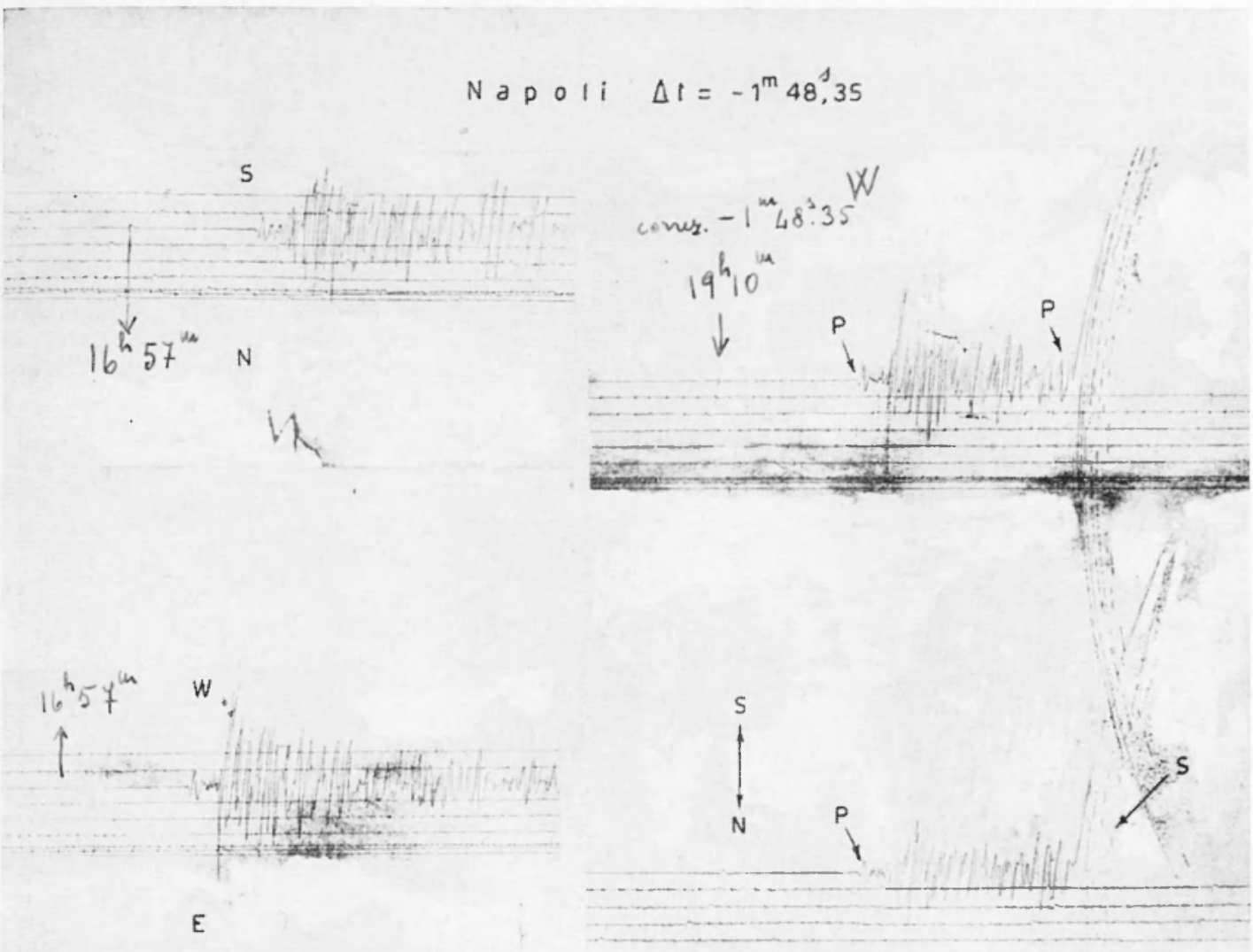

L'A quila $\Delta t=0,0$
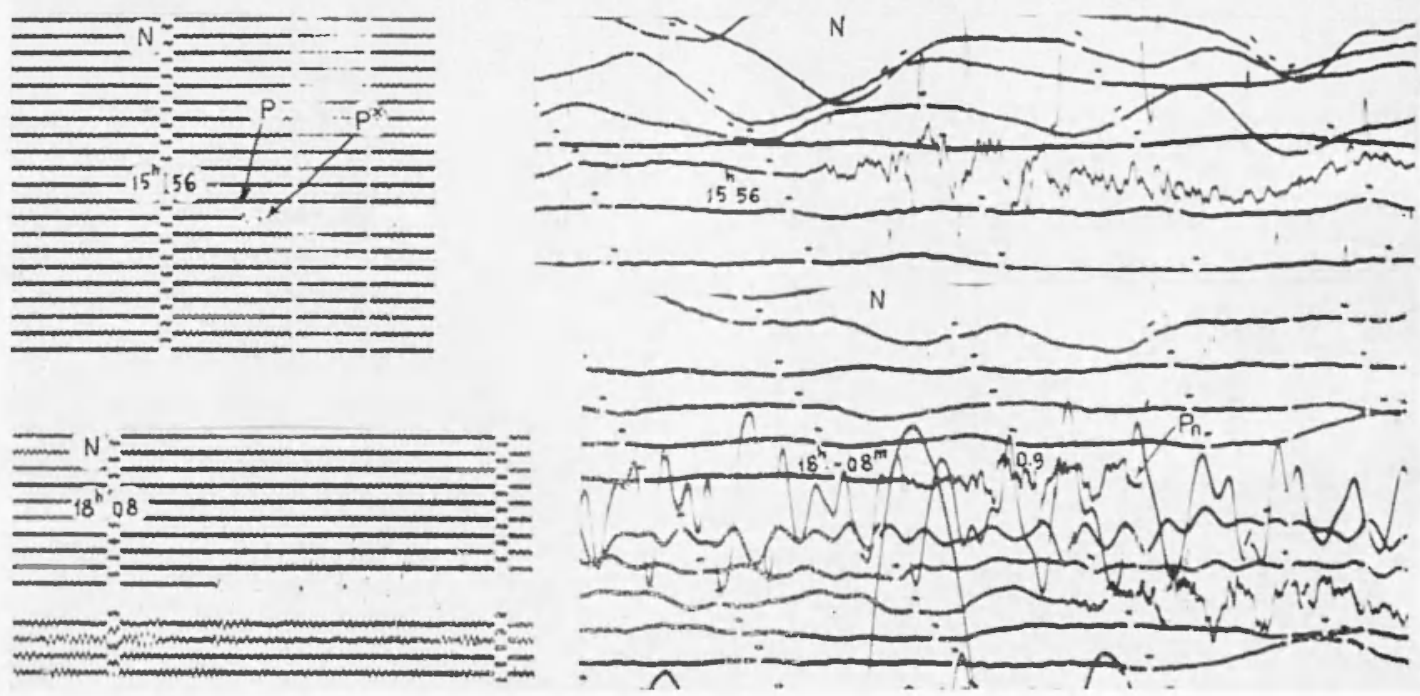
Padova $\Delta t=-1^{m} 45^{\mathrm{s}}$

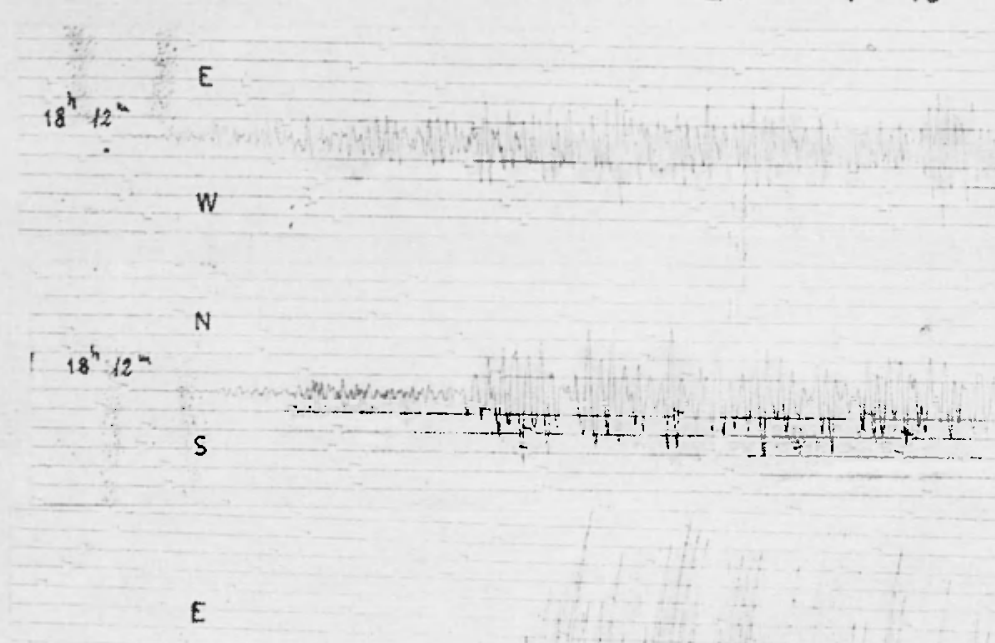

\section{$18^{\mathrm{h}} 22^{2 \mathrm{me}}$}

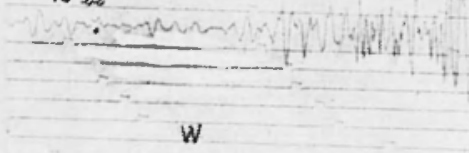

N

Wiechert $200 \mathrm{~kg}$.

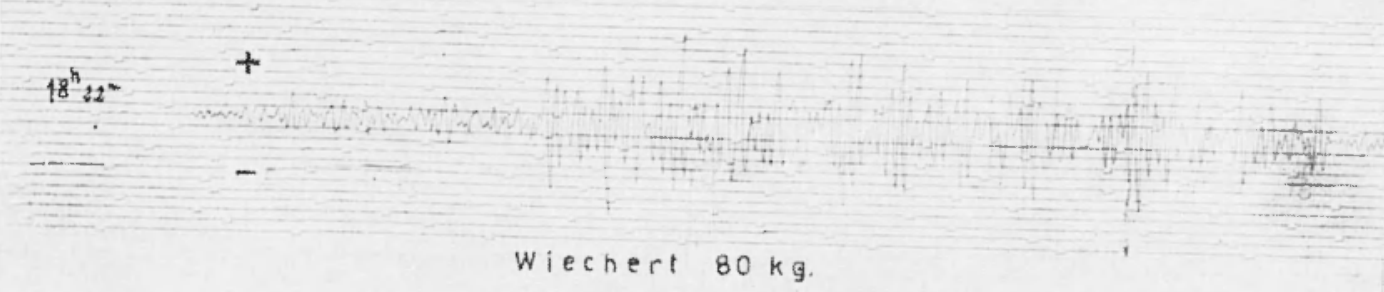




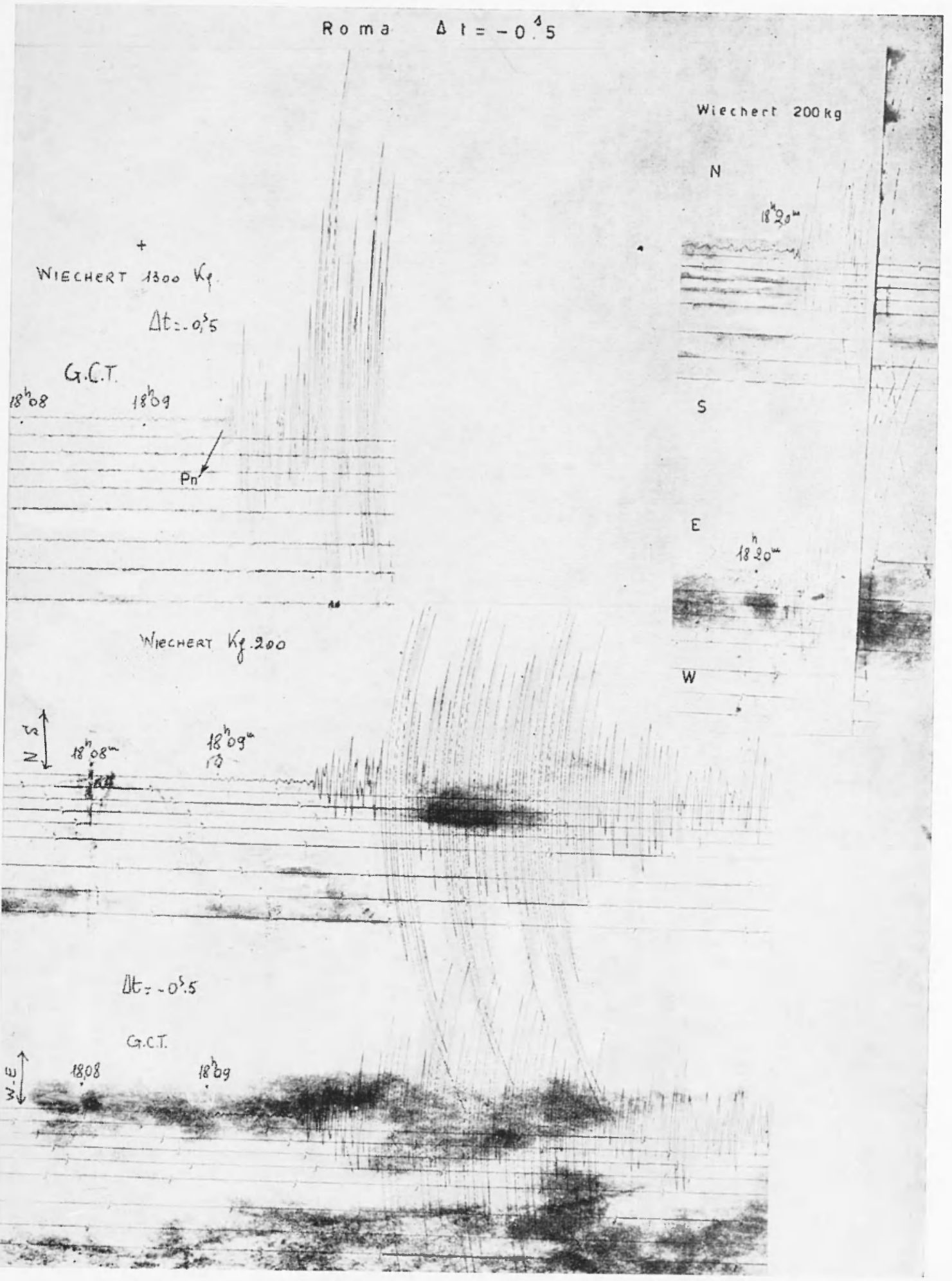


Rom a $\Delta t=-0.5$
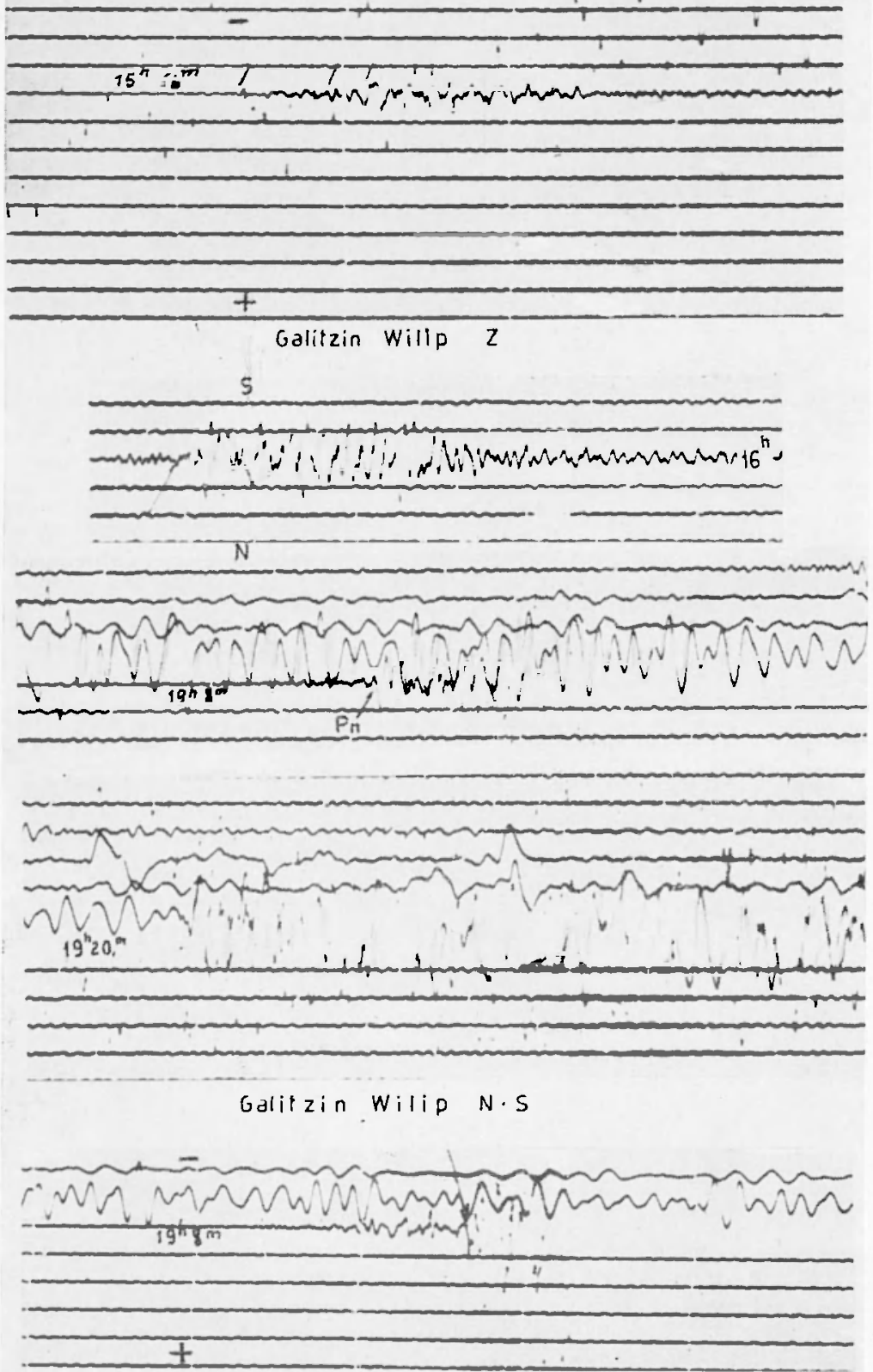

Galilzin wilip $z$ 
Rocea di Papa (Roma) $\Delta t=+1^{\Delta}$
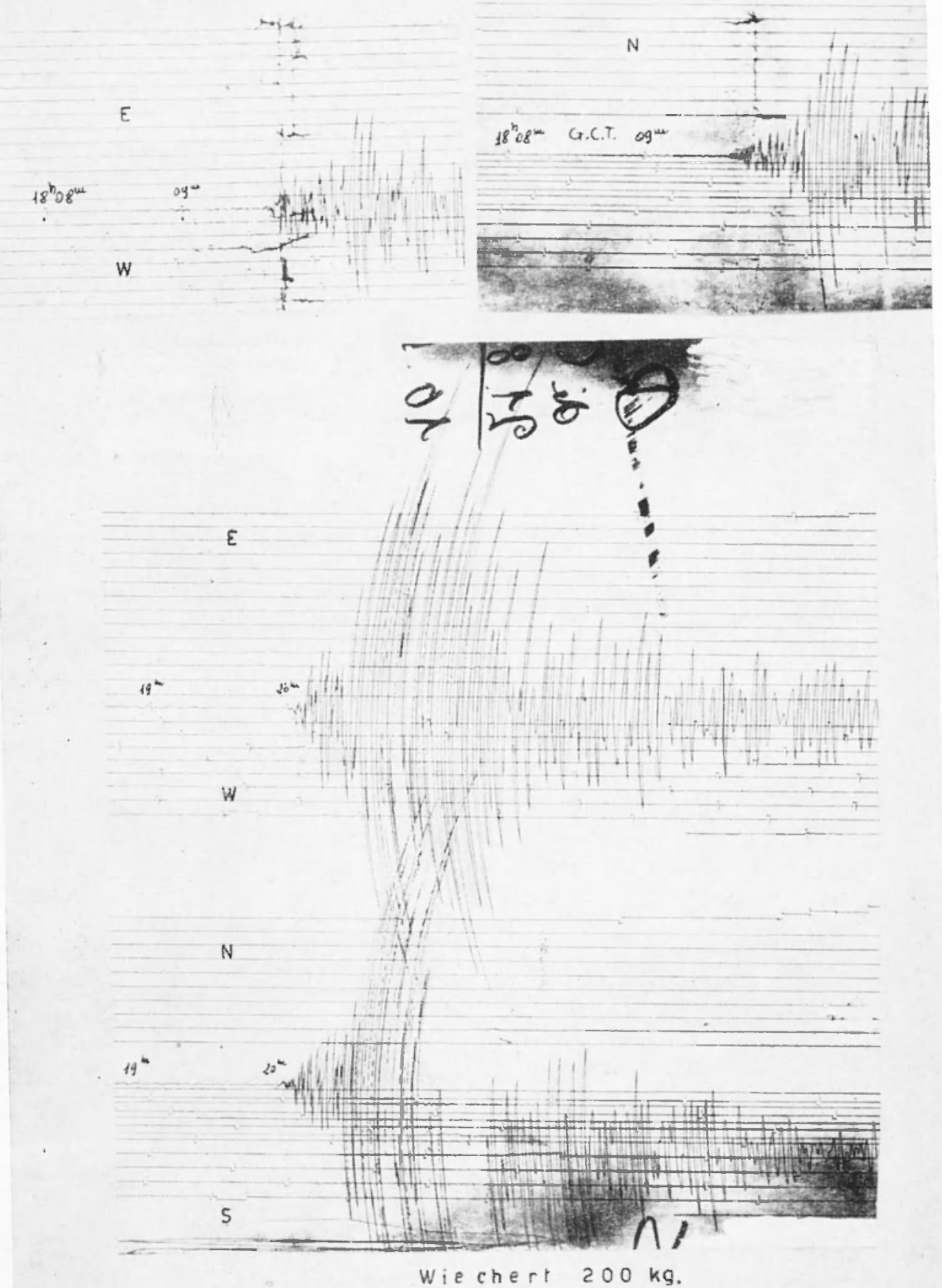
vajoni $\Delta=-2^{m} 25^{1}, 1$

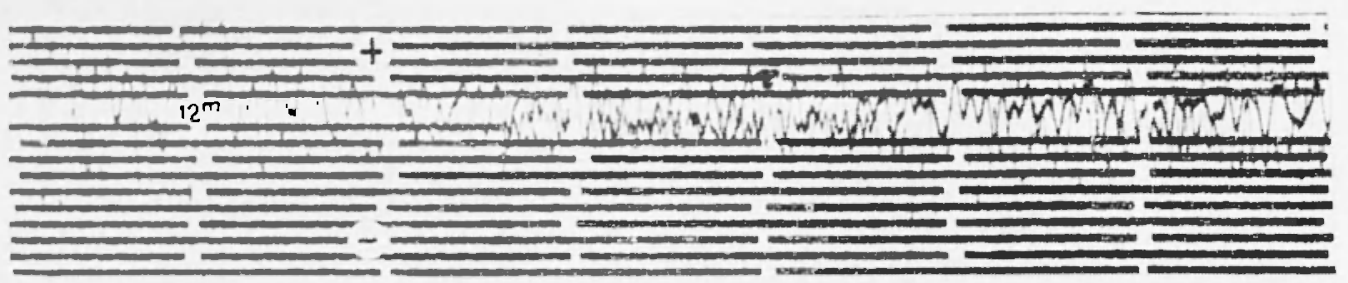

$+$

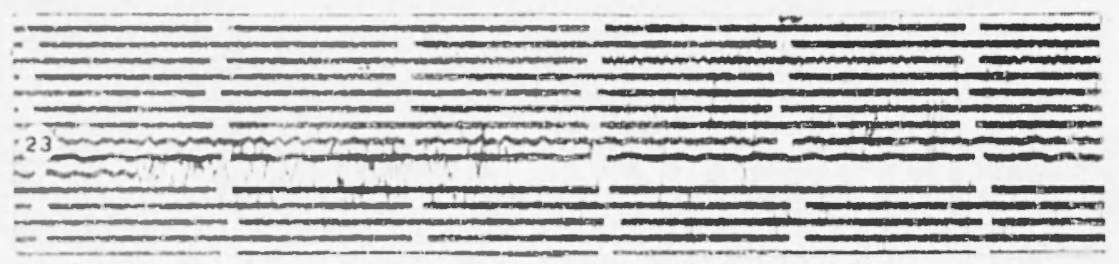

Verticale eleftromagneiico "Girianda"

$\operatorname{sicasourgo} A 1=+1,8$

$+$

1..ב-1 $18^{41}$ m ONב- - C C

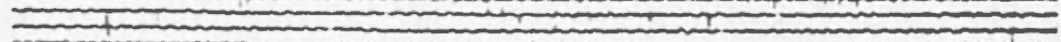
-

$18^{h} 21^{m}$

$5+2$

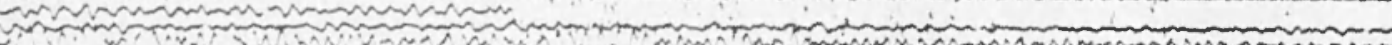

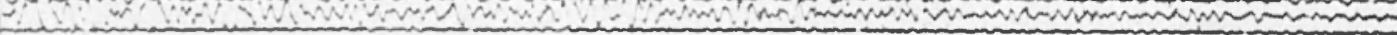
- E- 


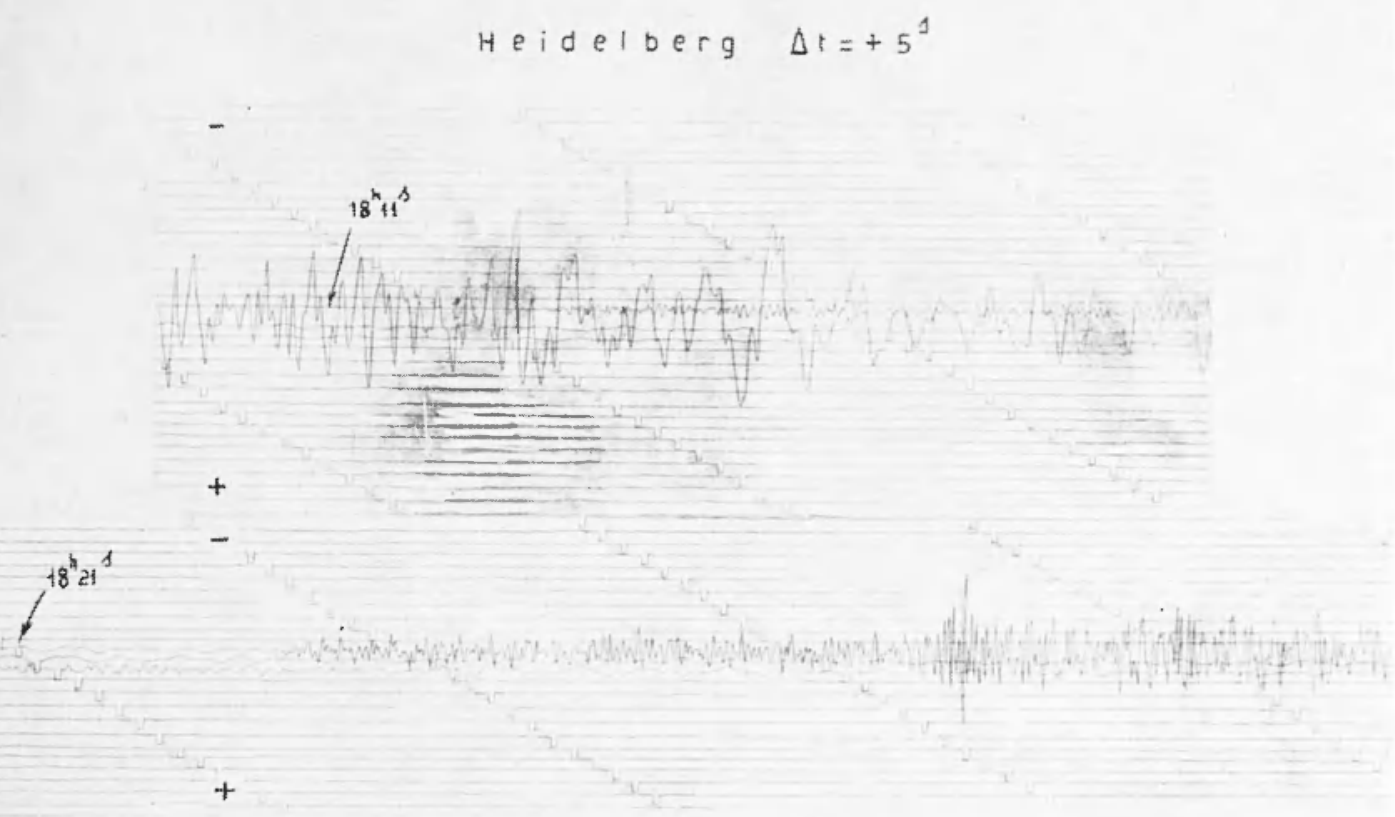

stoccarda $\Delta t=+4,9$
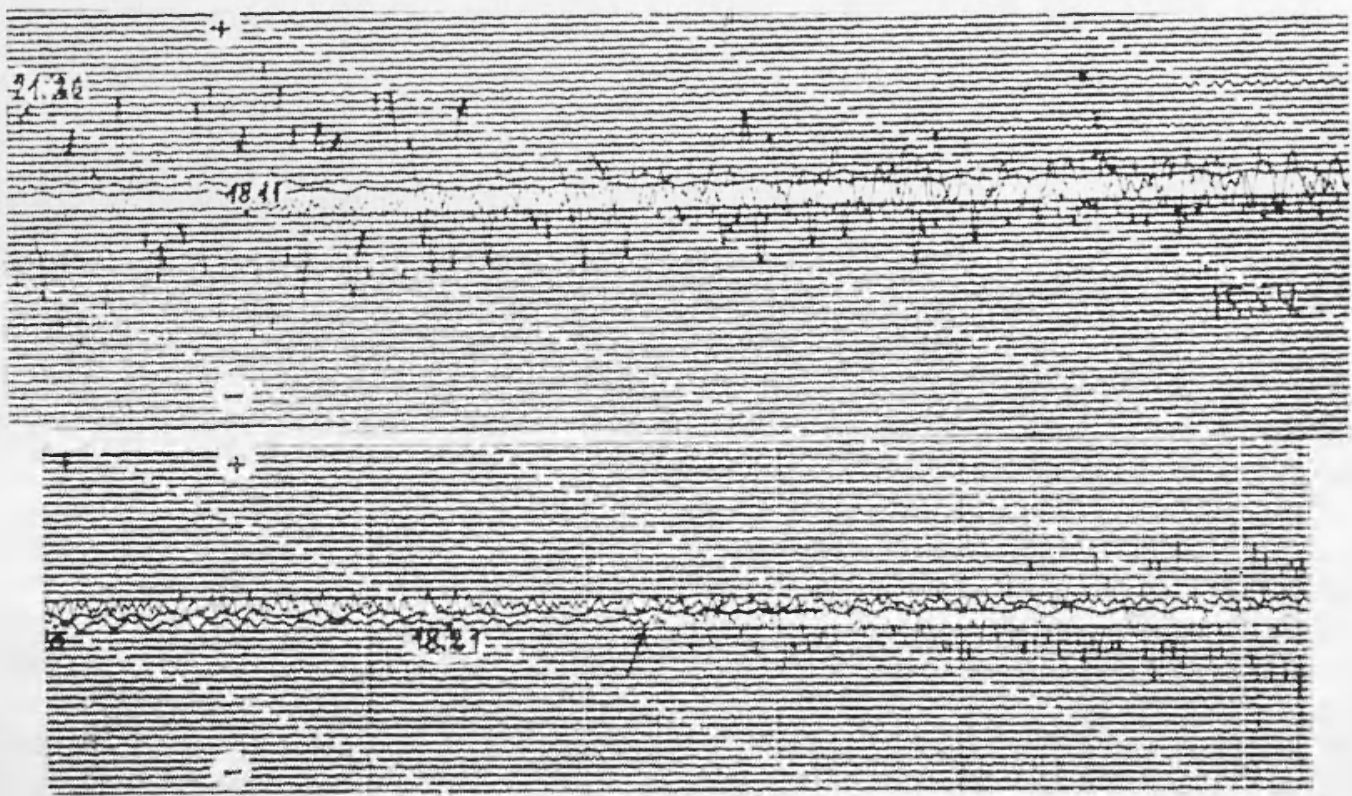


$$
\text { Ravensburg } \Delta t=+5^{s}
$$

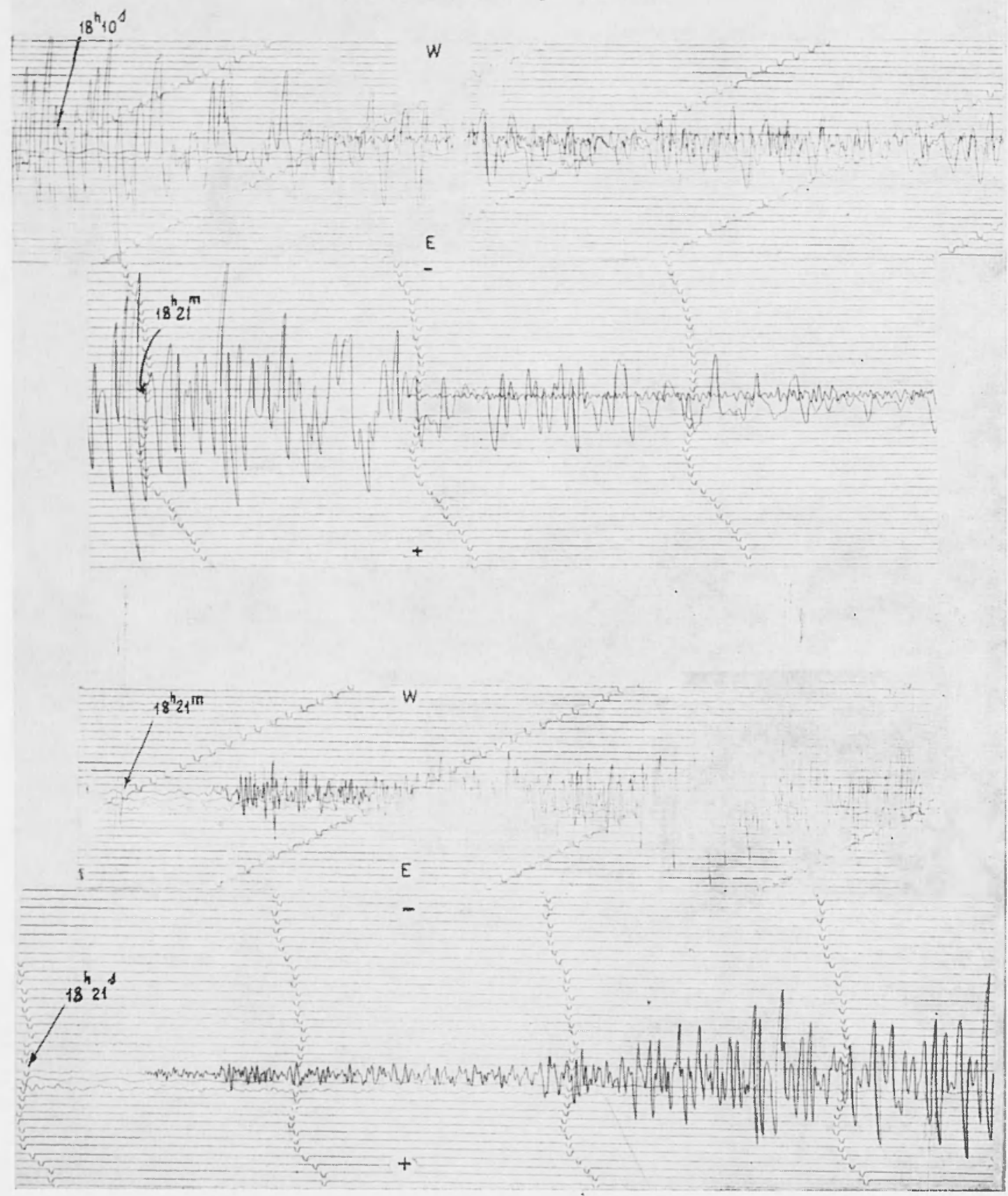


Tabella is

\begin{tabular}{|c|c|c|c|c|c|c|}
\hline \multirow{3}{*}{ Stayioni } & \multicolumn{3}{|c|}{ fa scosia } & \multicolumn{3}{|c|}{$2 a$ scossa } \\
\hline & \multirow{2}{*}{$\Delta(\lim )$} & \multicolumn{2}{|c|}{ tempo di tragito } & \multirow{2}{*}{$\Delta(\mathrm{km})$} & \multicolumn{2}{|c|}{ tempo di trasitto } \\
\hline & & $10^{\circ}$ impulso & $2^{n}$ impulsu & & $1^{\circ}$ impulso & 20 impulso \\
\hline Trieste & 508.692 & 695.01 & 715,81 & 509.000 & $69 *, 18$ & $73 * 78$ \\
\hline Zalgabria & 519.129 & 70,31 & & 520,462 & 70.28 & \\
\hline Vajont & $(009,0] 8$ & 81,11 & 85,71 & 608,203 & 81.28 & \\
\hline Pieve di Cadd. & 622.036 & 82.41 & & $(621,962$ & 82.28 & 86.18 \\
\hline Ravensburg & 853,703 & 112,11 & $117,3]$ & 852,925 & 112,48 & 116,78 \\
\hline Mestetten & 917.6992 & 119.51 & & 916,703 & 120,08 & 124.38 \\
\hline 'Tubinghen & $945,91: 5$ & 123,41 & $127,+1$ & 945,110 & $12+.08$ & 127.78 \\
\hline Stocearda & $963,+25$ & 125,21 & 129.51 & 962,814 & 125.78 & \\
\hline Strasburgo & $\mid(005,555)$ & 130,31 & | & $1004,59) 2$ & 130,88 & 8 \\
\hline Heidelberg & $10+1,480$ & 135,01 & 139,01 & 1040,888 & 134.98 & 139.88 \\
\hline Clermont $\mathbf{F}$. & 1092.4060 & & 147.61 & 1089.962 & $1+1,88$ & $1+7.98$ \\
\hline Uccele & 1351,426 & & 178,61 & 1350.0316 & 172,08 & \\
\hline Toledo & 1621.424 & 205,11 & & 1618,072 & $2(05,28$ & \\
\hline Malagra & 1753,795 & & 230.11 & 1750.202 & 220,88 & \\
\hline
\end{tabular}

piì probabile della longitudinale che segna l'inizio del sismogramma è clatá dall'oçuazione

$$
t_{1}=-(0,122788 \pm 0,000471) \perp+6,704017 \pm 0,149600
$$

che porta ad una velocità $v_{1}=8,14 \pm \pm 0,031 \mathrm{~km} / \mathrm{sec}$. Per il secondo impulso si è invere ottenuto

$$
t_{2} \cdots(0,126485+0,000767) 1+8,211113 \pm 0,87848
$$

(.)1 $v_{2}=7,906 \pm 0,048 \mathrm{~km} / \mathrm{sec}$.

Le analoghe dromocrone per la scossa delle ore $18^{\text {ni }} 19 \mathrm{~m} 29 \mathrm{~s}, 62$ somo invece

$$
\begin{aligned}
& \left.t_{1}=(0,122597 \pm 0,000571) .+\tau, 19193\right\rceil \pm 0,589013 \\
& t_{2}=(0,12 \bar{\tau} 600 \pm 0,001626) .1+\bar{\tau}, 81852 \bar{\tau} \pm 1,+5289 \overline{5}
\end{aligned}
$$

cui corrispondono le velocità

$$
v_{1}=8,175 \pm 0,037 \mathrm{~km} / \mathrm{sec} \text {. c } v_{2}=-7,837 \pm 0,099 \mathrm{~km} / \mathrm{sec} .
$$


Si noterà che i valori delle velocità per le due scosse sono praticamente coincirlenti e gli errori probabili molto piccoli, ciò evidentemento conferma l'attendibilità delle dromocrone. La velocità di 8,15 $\mathrm{km} / \mathrm{sec}$ ì ormai acquisita per le Pn e conferma il valore di $8,19 \pm 0,04$ di Di Filippo-Marcelli per il Gran Sasso, mentre la velocità ottenuta per i secondi impulsi (che nei sismogrammi sono particolarmente vistosi) trova conferma in molti ricercatori (Mohoroviçic, Jeffreys, Gutenberg, (aloi) e può attribuirsi alla zona di transizione táa la superficie di Molonoviçic e la parte superiore del mantello. A questo proposito ricordiamo che con sole quattro stazioni (Napoli, Aquila, Sorgenti del Peschiera, Roma) si era ottenuto una velocità di $7,8.43$ $\mathrm{km} / \mathrm{sec}$ per l'impulso che in tali stazioni più vicine all'ipocentro rappresenta l'inizio del sismogramma della scossa delle ore $15^{\mathrm{h}} 56^{\mathrm{m}}$.

La determinazione della natura fisica che all'ipocentro ha originato il terremoto delle ore $18^{\mathrm{h}} 19^{\mathrm{m}}$ è stata eseguita impiegando yli

'Tabella 6

\begin{tabular}{|c|c|c|c|c|c|c|c|c|}
\hline$N$ & Stazioni & $\begin{array}{l}\text { Na- } \\
\text { tura } \\
\text { lo im- } \\
\text { pulso }\end{array}$ & & $\Delta$ & & $\alpha$ & $\hat{i}$ & $\begin{array}{l}\cot \hat{i}= \\
=\operatorname{tg} \frac{1}{2}\end{array}$ \\
\hline$I^{\prime}$ & Napoli Ves. & + & $0^{\circ}$ & $38^{\prime}, 60$ & $237^{\circ}$ & $41^{\prime}, 60$ & $64^{\circ}$ & 0,48773 \\
\hline $2^{\prime}$ & Napolis. M. & + & 0 & 44,17 & 241 & 34,18 & 65 & 0,460331 \\
\hline $3^{\prime}$ & I'Aquila & + & 1 & 43,50 & 312 & 44,60 & 76 & 0,24933 \\
\hline $4^{\prime}$ & Taranto & 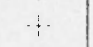 & 1 & 45,10 & 111 & 29,75 & 76 & 0,24933 \\
\hline $5^{\prime}$ & Peschiera & + & I & 58,10 & 307 & 41,39 & 79 & 0,19438 \\
\hline $6^{\prime}$ & Rolna & $-\frac{1}{1}$ & 2 & 05,50 & 293 & 16,84 & 80 & 0,17633 \\
\hline $7^{\prime}$ & Messina & - & 2 & 59,20 & 173 & 21,68 & 88 & $0.03+92$ \\
\hline $8^{\prime}$ & Fireuze & $\cdots$ & 3 & 51,25 & 313 & 41,60 & 89,5 & 0,00873 \\
\hline 9 & 'Trieste & $\therefore$ & 4 & 34,66 & 347 & 58,00 & $85, \tilde{5}$ & 0,07870 \\
\hline 10 & Vajont & + & 5 & 28,87 & 339 & 24.41 & 81,0 & 0.15838 \\
\hline 11 & Salò & -- & 5 & 32,83 & 324 & 36,92 & 80,6 & 0,16555 \\
\hline 12 & Pieve di C. & $(-)$ & 5 & 35,90 & 340 & 13,78 & 80,4 & 0,16914 \\
\hline 13 & Ravenshurg & + & 7 & 41,00 & 331 & 11,54 & 76,5 & 0,24008 \\
\hline 14 & Mestetten & 1 & 8 & 15,70 & 330 & 15,65 & 75,7 & 0,25490 \\
\hline 15 & 'T'ubinghen & - & 8 & 30,76 & 331 & 50,35 & 75,5 & 0,25862 \\
\hline 16 & Stocearela & - & 8 & 40,25 & 333 & 12,19 & 75 & 0,26795 \\
\hline 17 & Strasburgo & - & 9 & 03,00 & 327 & 27,12 & 74,8 & 0,27169 \\
\hline 18 & II eiclelberg & + & 9 & 22,11 & 333 & 33,41 & 74,1 & $0,28+86$ \\
\hline 19 & licele & -1 & 12 & 09.77 & 325 & 56,96 & 70,1 & 0,36199 \\
\hline 20 & Tolerlo & 一 & 14 & 35,57 & 269 & 00,00 & $6(j, \tilde{5}$ & 0,43481 \\
\hline 21 & Malaga & $\cdots$ & 15 & 47,05 & 260 & 02,20 & 64 & 0,48773 \\
\hline
\end{tabular}


impulsi iniziali rilevati in 21 stazioni (tab. 6) e seguendo il noto metodo di Byerly esteso da I)i Filippo per tener conto della profondità ipocentrale. La posizione delle stazioni distese sulla sfera ipocentrale e la sua proiezione stereografica polare sono state ottemute impiegando le tabelle da noi grià pubblicate in occasione di uno studio eseguito sui terremoti di frattura delle Alpi Orientali.

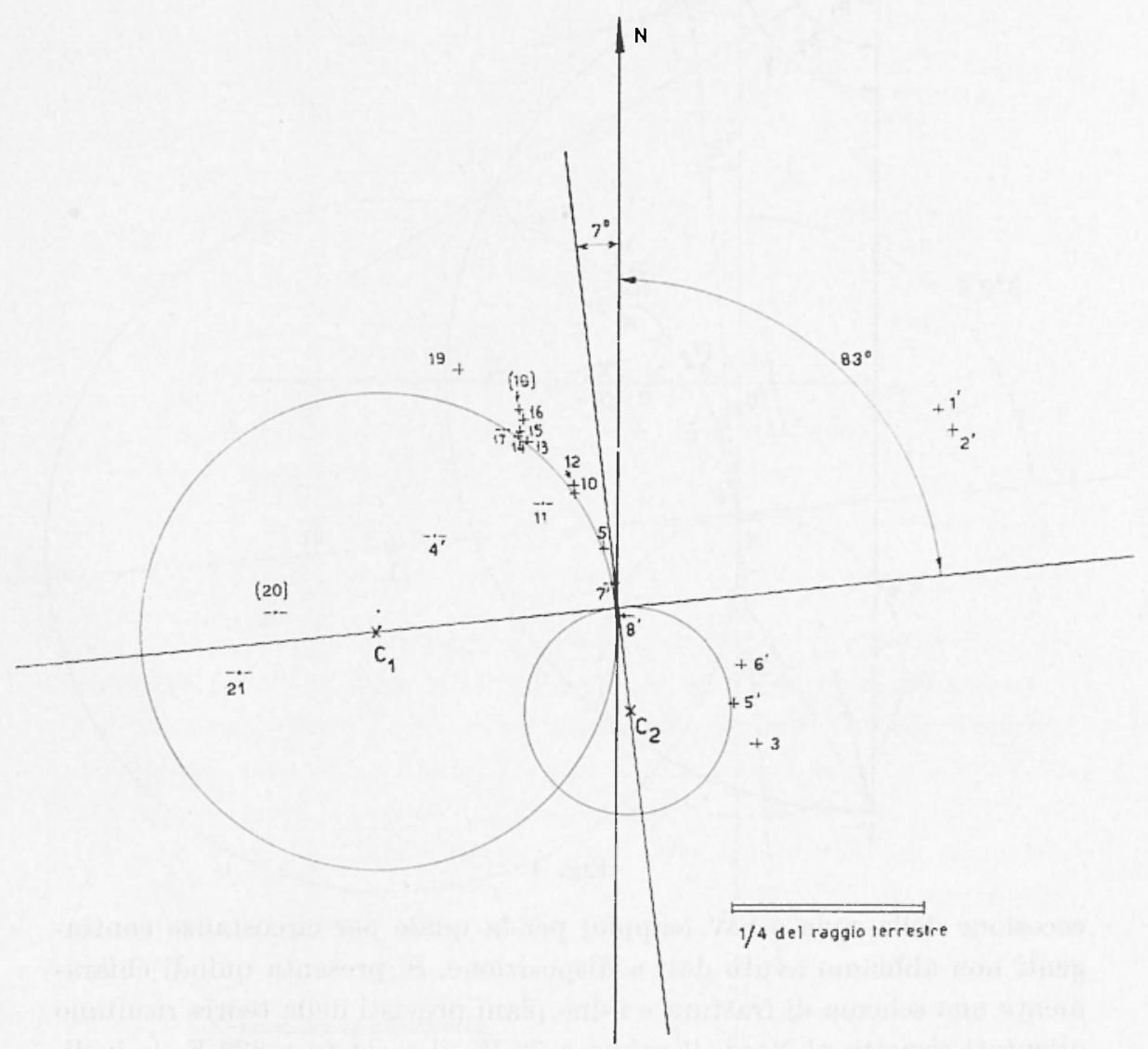

Fig. 3

Le stazioni i cui numeri sono segnati da un indice sono state riportate impiegando il raggio opposto, dato che il diretto formava con il raggio terrestre un angolo minore di $90^{\circ}$ e quindi non intersecava la sfera ipocentrale. La Fig. 3 rappresenta la posizione delle stazioni distese proiettate sul piano equatoriale dall'mtipocentro. Il cerchio $\mathrm{C}_{1}$ è delimitato chiaramente in quanto deve separare le dilatazioni $\tau^{\prime}$ e 17 dalle 
rompressioni 9, 10, 13, 14, mentre il rerchio $\mathrm{C}_{2}$ normale al precedente risulta determinato con buona approssimazione dalla compressione $5^{\prime}$ e dalla dilatazione 8'. I due cerchi dividono il piano proiezione in quattro zone le cui opposte sono caratterizzate da segni dello stesso tipo, ad

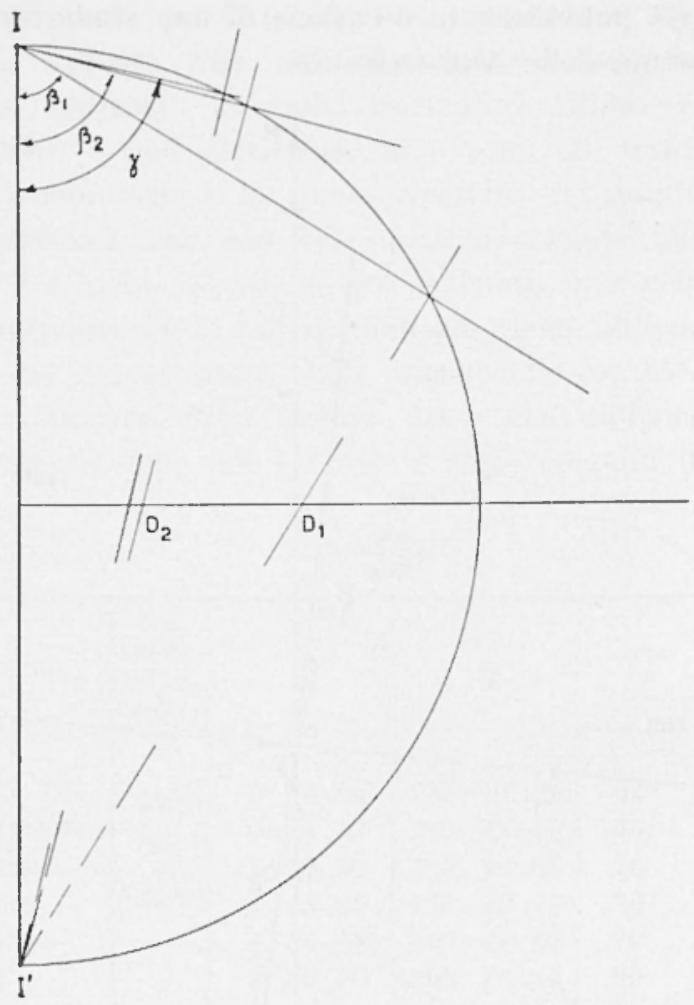

Fig. 4

ecrezione della zona a SW (cappio) per la quale per circostanze contingenti non abbiamo avuto dati a disposizione. Si presenta quindi chiaramente uno schema di frattura a i due piani previsti della teoria risultano orientati rispetto al Nord, il primo a $7^{\circ} \mathrm{W}$, il secondo a $83^{\circ} \mathrm{E}$; le inclinazioni rispetto al raggio terrestre sono nell'ordine $\bar{p}_{1}=58^{\circ}, 5 ; \hat{\rho}_{1}=75^{\circ}$ (Fig. 4). Il prolungamento dell'asse padicale dei due cerchi forma con il Nord l'angolo di $18^{\circ} \mathrm{E}$ ad è inclinato rispetto al raggio terrestre per l'ipocentro dell'angolo $y=77^{\circ}, 5$; ne segue che le coordinate polari rispetto all'epicentro della intersezione della retta comune ai due piani con la superficie terrestre (polo) sono $a=18^{\circ} \mathrm{E}, \varrho=1^{\circ} 53^{\prime}$.

Anche la scossa delle $18^{\mathrm{h}} 19^{\mathrm{m}}$ ha presentato uno schema di frattura rome compare in Fig. 5 ottenuta ron gli elementi riassunti in tab. 5 . Si 
noterà che anche in questo caso il cerchio $C_{1}$ is individuato con grandissima precisione dalle compressioni $6,8,14$, e dalle dilatazioni 4 ', 11, 13,

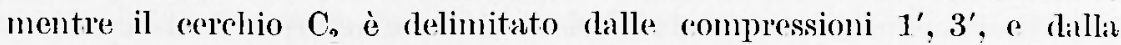
dilatazione $5^{\prime}$.

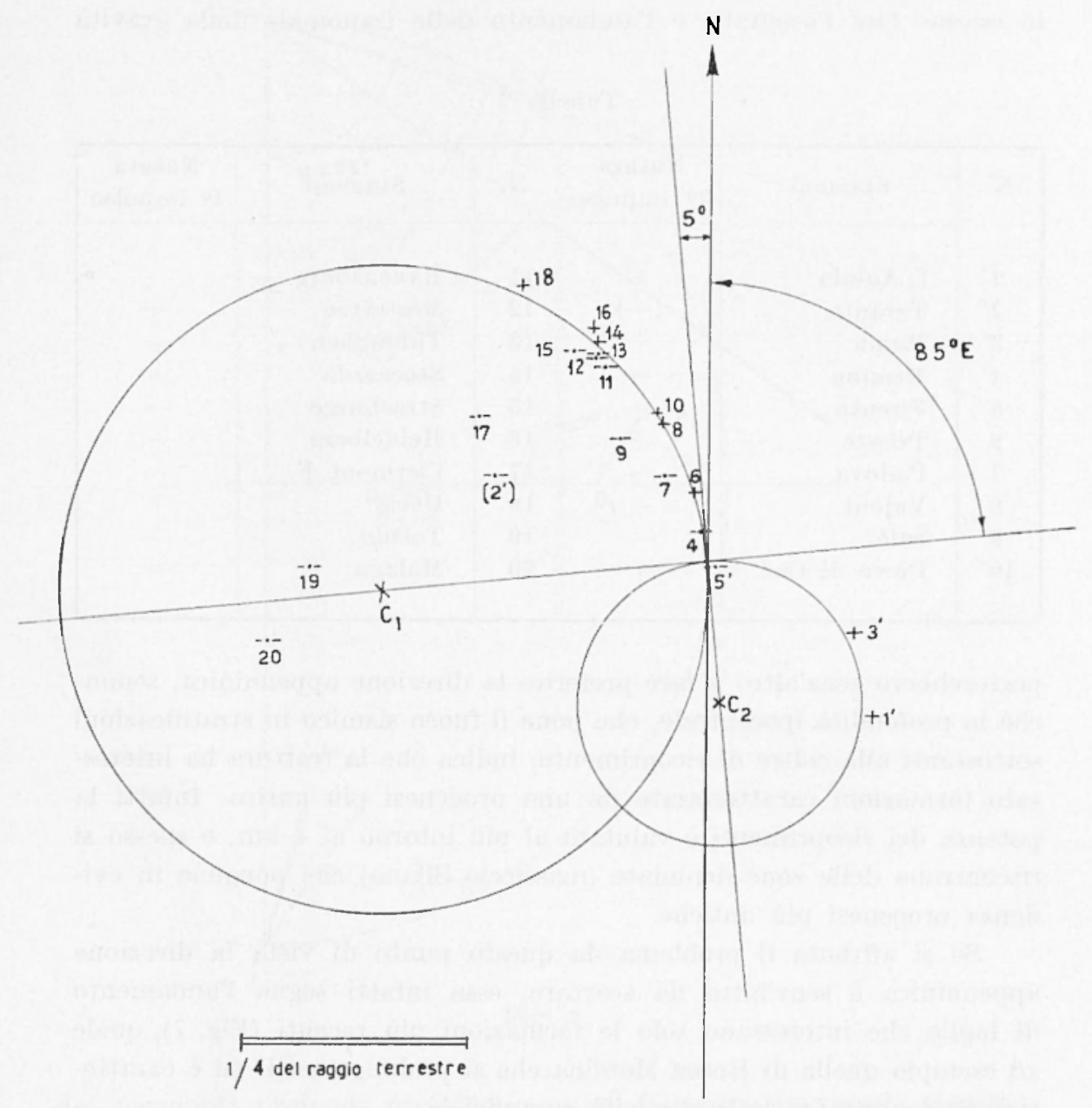

Fig. 5

I due piani formano con il Nord gli angoli di $5^{\circ} \mathrm{W}$ a $85^{\circ} \mathrm{E}$ e le loro inclinazioni rispetto al raggio terrestre sono $\beta_{\mathrm{t}}=55^{\circ}, \beta_{2}-73^{\circ}$. Ia retta intersezione dei due piani forma con lo stesso raggio l'angolo di $75^{\circ}$ el infine il polo ha rispetto all'epicentro coordinate $\alpha=19^{\circ}, 5, \varrho=1017^{\prime}, 5$. (Fig. 6). I risultati ottenuti per le due scosse sono quindi praticamente coincidenti se si tiene conto dell'approssimazione grafiea e della diffe- 
renza che intercorre tra le posizioni dei due ipocentri. Come è noto lis teoria lascia indeterminata la direzione dell'effettivo piano di frattura, che va scelto tra i due ottenuti dal metodo, in base a considerazioni di natura tettonica, orografica o ad elementi riguardanti la sismicità della zona in esamo. Ora l'orografia e l'andamento delle isanomale della grravità

Tabella 7

\begin{tabular}{|c|c|c|c|c|c|}
\hline$x$. & Stazioni & $\begin{array}{l}\text { Natura } \\
\text { to impulso }\end{array}$ & $N$ & Stazioni & $\begin{array}{l}\text { Natura } \\
\text { 10 impulso }\end{array}$ \\
\hline $1^{\prime}$ & JiAquila & + & 11 & Ravenshurg & - \\
\hline $2^{\prime}$ & Taranto & $(-)$ & 12 & Mestetten & - \\
\hline $3^{\prime}$ & Roma & + & 13 & 'Tubinghen & - \\
\hline$t^{\prime}$ & Messina & - & 14 & Stocearda & t \\
\hline$\tilde{5}^{\prime}$ & Firenze & 一 & 15 & Strashurgo & - \\
\hline 6 & 'l'rieste & $\therefore$ & 16 & Heidelberor & + \\
\hline 7 & Padova & $\ldots$ & 17 & ('lermont F. & - \\
\hline 8 & Vajont & + & 18 & liecle & + \\
\hline 9 & Salò & - & 19 & Toledo & — \\
\hline 10 & Pieve di Cad. & -+ & 20 & Malaga & - \\
\hline
\end{tabular}

porterebbero senz'altro a fare preferire la direzione appenninica, senonché la profondità ipocentrale, che pone il fuoco sismico in stratifieazioni sottostanti alla coltre di ricoprimento, indica che la frattura ha interessato formazioni caratterizzate ca una orogenesi più antica. Infatti la potenza dei ricoprimenti è valutata al più intorno ai $4 \mathrm{~km}$, e spesso si riscontrano delle zone denudate (massiccio Silano) che pongono in evidenza orogenesi più antiche.

Se si affronta il problema da questo punto di vista la direzione appenninica è senz'altro da scartare, essa infatti segna l'andamento di faglie che interessano solo le formazioni più recenti (Fig. 7), quale ad esempio quella di Rocea Monfina che si prolunga a SE ed è caratterizzata da una not'vole sismicità superficiale.

Riferendoci alle formazioni più antiche la tettonica e l'osservazione hammo posto in evidenza tutta ma serie di faglie profonde con andamento trasversale rispetto alle precedenti. Due delimitano il promontorio del Gargano, una è molto prossima alla zona epicentrale. In particolare quest'ultima è visibile anche in superficie, marcatamente ai suoi estremi (coste meridionali della Penisola sorrentina e Valle dell'Ofanto) e presenta trace visibili anche se non rettilinee nella parte intermedia di 
ricoprimento. Tale sistema trasversale ben si accorda con quanto abbiamo già posto in evidenza a proposito della sismicità del Meditermaneo meridionale e in particolare del basso Tirreno, ore i piani di frattura

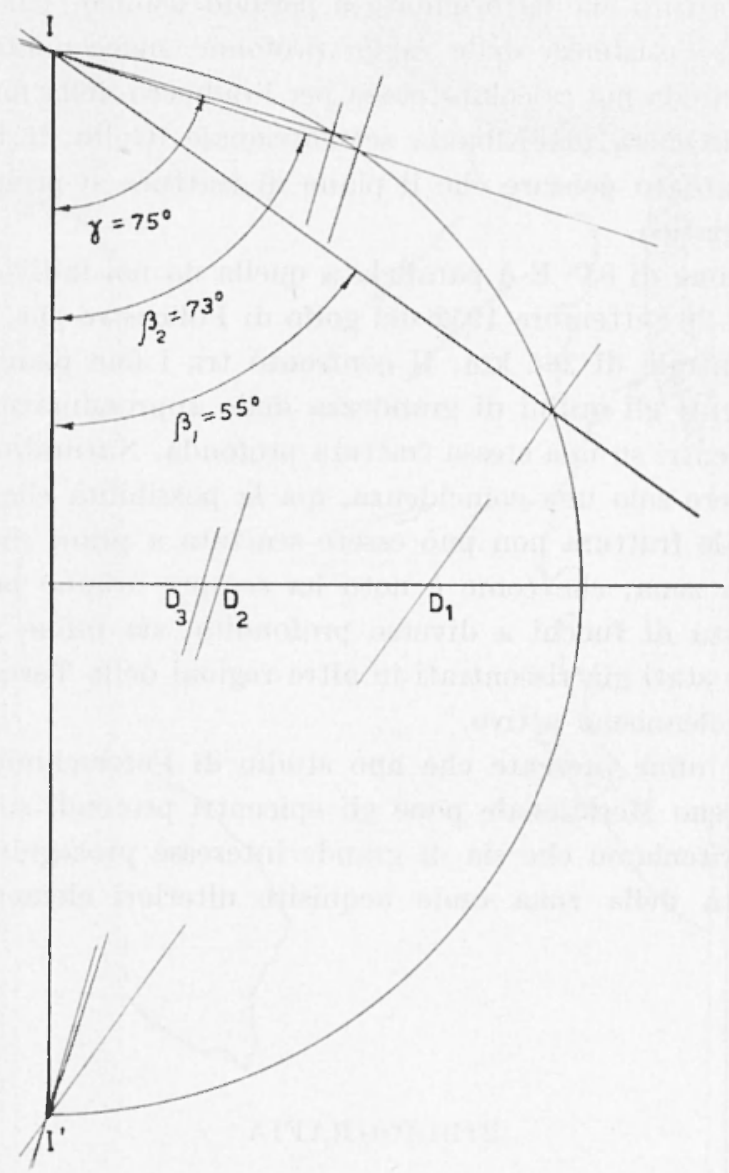

Fig. 6

dei terremoti profondi presentano un andamento trasversale rispetto alla Penisola italiana, andamento segnato dai rilievi vuleanici che sono il supporto delle isole Lipari, a loro volta parallele alla supposta faglia africana.

Lo stesso Appennino centromeridionale viene dai più considerato una antica dorsale sottomarina coronata da un vuleano: il Vulture, e separata dalla Calabria che non presenta alcun rapporto tettonico con l'Appennino. 
Ritomando ora alle due scosse da noi studiate, si nota che le dire. zioni dei piani orientati a $83^{\circ}, 85^{\circ}$ Es semono tale allineanento trasversale per cui ritrolamo che essi (praticamente concidenti) siano l'eftettivo piano drellat frattura che ha orieginato il periodo sisminco. Thale scelta non solo ronforma l'essistonza delle facrlie profonde suaceenmate, ma dato

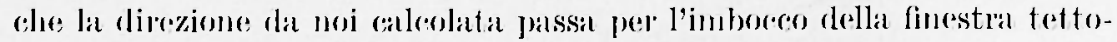
nicar rhe si riscontma ncll'Albania settentriomale (Golfo di Drin) non è del tulto azzardato pensate che il piano di frattum si prolunghi al disollo dell'drantion.

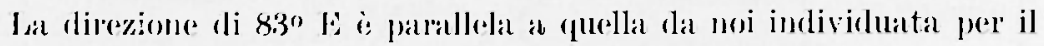

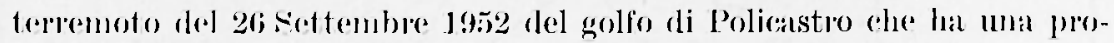
fondita iposentrale di 26.1 knt. Il confronto tra i due piani, quando si tongano presenti grli ordini di grandezza delle approssimazioni grofiehe, porta gli ipocentri su uma stessa fratluma pofofonda. Natumalmente questo fatto puo essere solo man rooncidenza, ma la possibilita che esista realmente matale fäthura non puo cessere scatata a priori sia per il rulcanismo della zona, che come is noto ha sempere origine profoncla, sia per la presenza di fuochi a diverse profondità, sia infine perehé fatti analoghi sono stati già risentati in altre regioni della Terra calatterizzate da un vulounismo attivo.

Tenendo infine presente che nuo studio di Peterschmitt sui terremoti dol Tirreno Meridionale pone gli epicentri profondi su una superficic ronica, riteniamo ehe sia di grande interesse proseguire l'indagine della sismiceita della zoma onde acequisioe ulterioni elementi alla suat lattonicas.

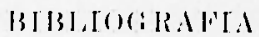

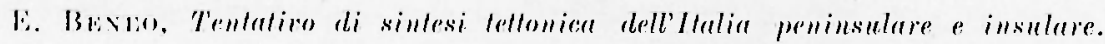
"Boll. Sore. Cecologrima Italianal", LXVIII, (1949).

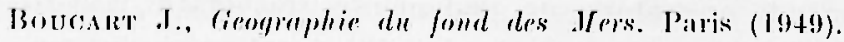

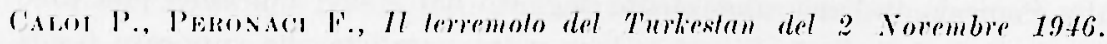
"Amali di Cerofision". I, (19+8).

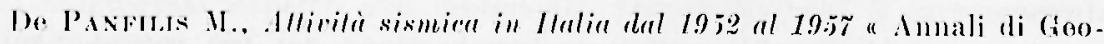
fisje:a ", XII. (19:59).

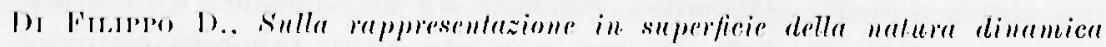

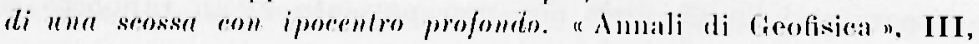
(19:50). 
D. DI PIIIPO - F. PERONACI

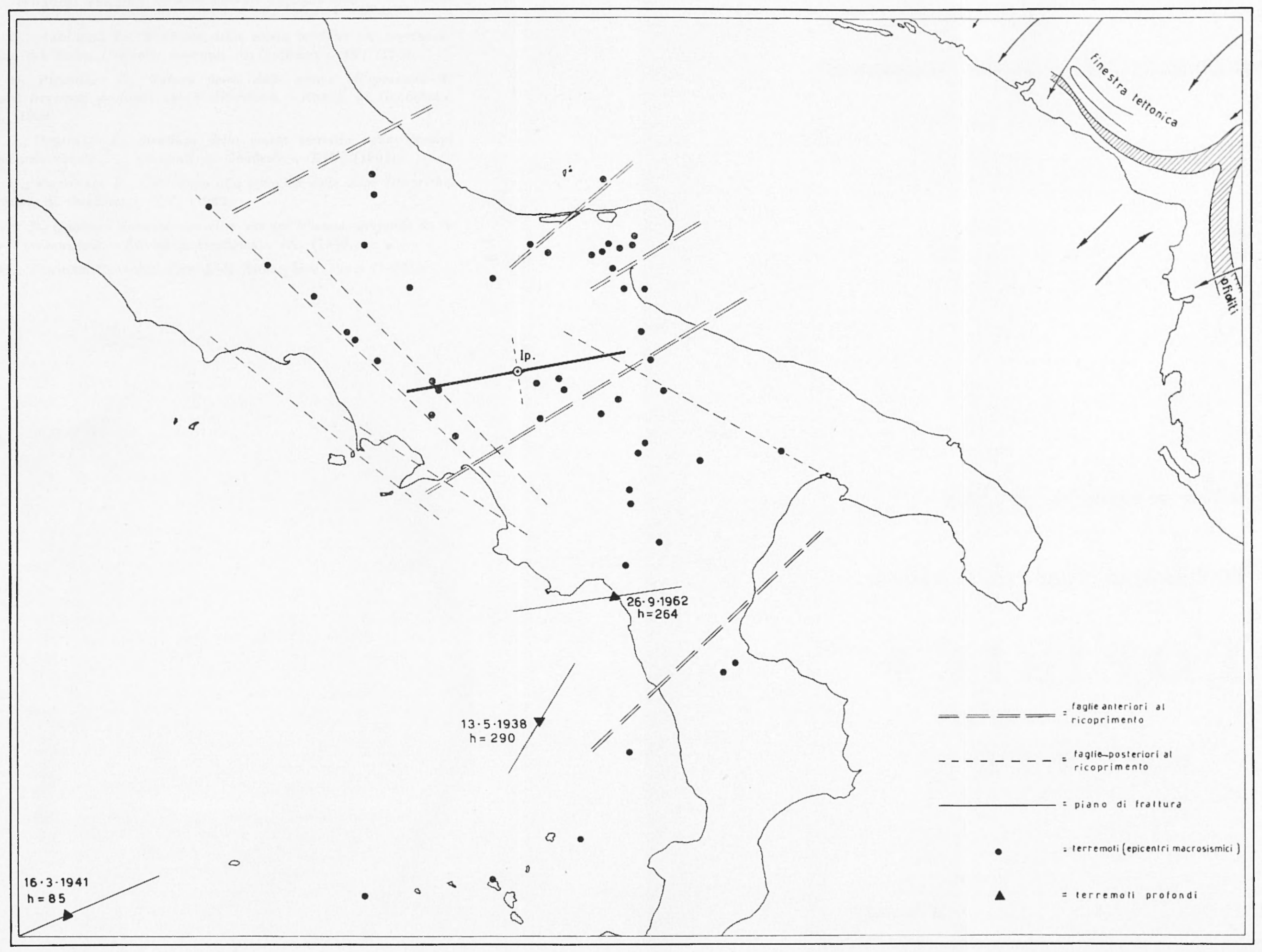

Fig. 7 
Di Filippo D., Marceidi J., Strullura della crosta terrestre in corrispondenza dell'Italia Centrale. "Annali di Cieofisiea ", IV, (1951).

Di Filippo D., Peronaci F., Natura fisica della scossa allipocentro di alcuni terremoti profondi nel Mediterraneo. "Annali di Geofisica ", XII, (1959).

Di Filippo D., Peronaci F., Struttura della crosta terrestre nelle Prealpi Lombardo-Venete ... "Annali di Geofisica", XIV, (1961).

Di Filippo D., Peronaci F., Contributo alla tettonica delle Alpi Dinariche. "Annali di Geofisica", XV, (1962).

Peterscimite S., Quelques données nouvelles sur les seismes profonds de lu mer Tyrrhenienne. "Annali di Geofisica ", IX, (1956).

Thermina It., Tenmer C., Levolution de la Tithosphère. Paris (1957). 